\title{
Unidirectional compression of electrorheological fluids in electric fields
}

\author{
Yonggang Menga) \\ State Key Laboratory of Tribology, Tsinghua University, Beijing 100084, China \\ Frank E. Filisko \\ Department of Materials Science and Engineering, The University of Michigan, Ann Arbor, Michigan 48105
}

(Received 21 February 2005; accepted 17 August 2005; published online 3 October 2005)

\begin{abstract}
A series of unidirectional compression tests on electrorheological fluids has been carried out with different plate sizes, initial plate separations, and applied voltages. Experimental results of compression pressure were compared with the continuum non-Newtonian squeeze-flow theory in a normalized form. It was found that the compression resistance of the electrorheological fluid in an electric field was much higher than that predicted by the theory when the fluid was compressed to some extent and/or when the initial plate separation was relatively small. Characteristics of the pressure variation with the reduction in electrode gap width have been analyzed, and the compression behaviors under different experimental conditions have been discussed in terms of the morphology of particle aggregations under compression. () 2005 American Institute of Physics.
\end{abstract} [DOI: $10.1063 / 1.2064310]$

\section{INTRODUCTION}

When a strong electric field is applied on some kinds of dispersions composed of micrometer-sized dielectric particles and insulating oils, the randomly dispersed particles form aggregated structures bridging between the electrodes as a result of polarization. ${ }^{1}$ This change in the microstructure of the particle distribution can result in a dramatic and reversible change in mechanical behaviors of the dispersions under shearing, tension, and compression. ${ }^{2}$ During the past two decades, a lot of efforts have been made worldwide for understanding the structure-property relationship in the fieldinduced transition. Moreover, many studies for demonstrating great potential for industrial applications of the so-called electrorheological effect were conducted. At the present, most of the researchers believe that it is the particle structure formed in electric fields that determines the mechanical strength of such dispersions. However, there is still an argument about what particle structure plays the predominating role in response to mechanical loading.

Several models have been proposed from the simple single chain and body-centered tetragonal lattice to the lamellar structure. ${ }^{3}$ In the recent study conducted by one of the authors, it has been shown that during shearing of electrorheological fluid samples, the particles rearrange from unorganized distribution of columns into tight packed lamellar formations consistent with the shear field. ${ }^{4}$ Obviously, the lamellar structure is stronger than the unorganized columns to support a shearing force in the direction parallel to its plane. This finding implies that the electric field is not the sole extrinsic factor that determines the morphology of particle structures. The history of mechanical loading is also involved in the development.

Not only shear stress but also compressive stress could impact on the state of particle aggregations, which are pri-

\footnotetext{
a) Author to whom correspondence should be addressed; electronic mail: mengyg@mail.tsinghua.edu.cn
}

marily induced by an electric field. The previous work concerning compression behaviors of electrorheological fluids could be classified into two categories. One is on oscillatory compressive squeeze flow, where the change in distance between the electrodes confining fluid samples is usually only a few percent, while the squeezing velocity could reach a high value if the oscillatory frequency is high (see, e.g., Refs. $5-7)$. The other is on unidirectional monotonous compression, where the reduction in the electrode gap could be to a large extent, but the squeezing velocity is often limited to a lower value (see, e.g., Refs. 8-10).

Tao et al. ${ }^{11}$ reported that the static yield shear stress of electrorheological fluids increased greatly after the fluids were compressed along the field direction, similar to the behavior of magnetorheological fluids. ${ }^{12}$ They attributed the enhancement of shear strength to thicker particle columns produced by the compression.

To predict the compressive resistance of electrorheological fluids in electric fields, a continuum non-Newtonian fluid theory, assuming electrorheological fluids to be biviscous media with a field-strength-dependent yield shear stress, has been developed and successfully used to model oscillatory compression experiments with a reasonable accuracy. ${ }^{5}$ On the other hand, all of the few published works on unidirectional compression just released some experimental results. None of them discussed the question of whether the same theory is suitable to deal with the unidirectional compression problem, excluding the very recent work done by Tian $e t$ $a l .{ }^{13}$ which recognizes that the theory underestimates the compressive resistance when the electrode gap separation is small. In this case, there is still a lack of detailed experimental and theoretical investigations on compression behaviors of electrorheological fluids as well as insight into the relationship between the compression behavior and particle aggregation structures.

In this paper, quasistatic unidirectional compression experiments, in a series of different electric-field intensities 


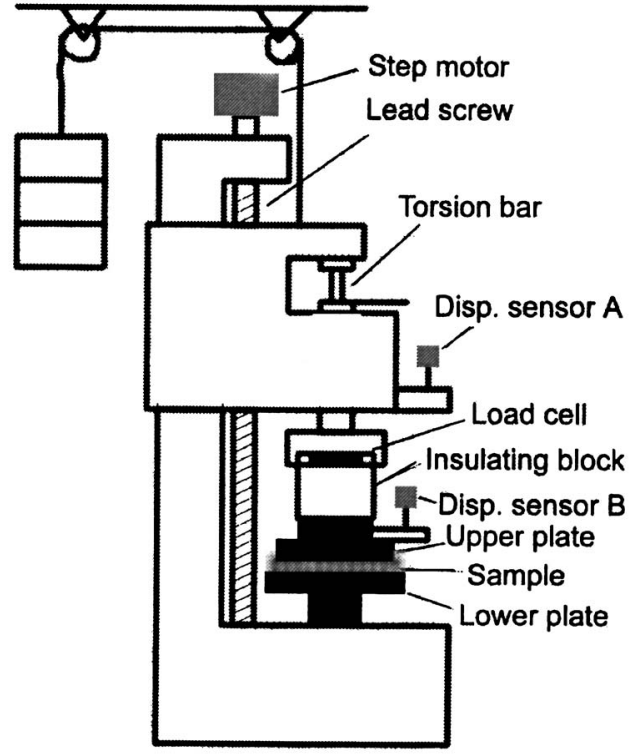

FIG. 1. Sketch of the modified rheometer used for compression test.

(electrode sizes and initial electrode gap widths), have been done on a Weissenberg-type rheometer which has been specially modified for the purpose of this study. The obtained experimental results have shown that compression in electric fields can always make electrorheological fluids so strong that they behave like consolidated granular materials with extremely high load supporting capability, provided that some critical conditions, including the electrode gap width and the applied voltage, are satisfied.

\section{EXPERIMENTAL METHODS}

To perform compression tests, a step motor-lead screw loading unit was added onto a Weissenberg rheometric instrument, together with one load cell (Entran ELW-D1-100L) and two linear variable differential transformer (LVDT) displacement sensors (see Fig. 1). The sample to be tested was filled out in the gap formed between the flat lower steel electrode, which can rotate at a preset speed around the vertical axis, and the upper steel electrode, the position of which can be changed along the vertical axis by the loading unit.
Before compression, a dc voltage was imposed on the sample and was kept constant during the whole period of compression. The normal force and the torque, which were generated when the lower electrode was rotating, acting on the upper electrode, were measured, respectively, with the load cell and a torque sensing unit which is composed by a torsion bar connecting with an armature of a LVDT linear sensor. Meanwhile, the variable positions of the upper electrode and the moving head were detected with displacement sensor B and displacement sensor A, respectively (see Fig. 1).

The reason for measuring the movements of both the upper electrode and the moving head independently is for taking account the elastic deformation of the polyoxymethylene insulating cylinder, inserted between the load cell and the upper electrode. The deformation arises under the high normal force, possibly generated during compression tests. In addition to the elastic deflection of the upper electrode, the lower plate also moves down slightly under high normal forces as a result of elastic deflection of the supporting part, the amount of which was estimated to be about one-fifth of the deflection of the upper plate by a separate measurement.

The true change of the electrode gap width during compression was obtained from the displacements of both the upper and the lower plates. Particular attention was paid to the influence of the clearance existing in the screw connection between the lead screw and the moving head, and a counterweight was applied through a pulley to eliminate the clearance and to ensure continuous movement of the moving head during test.

The electrorheological fluid sample used in the experiments consisted of $\mathrm{NaY}$ zeolite particles and silicon oil with a particle concentration of $42 \%$ in weight (volume fraction of $27 \%$ estimated from the densities of the solid and liquid phases). This kind of zeolite dispersions has a relatively strong electrorheological effect, the yield shear stress of which exceeds $12 \mathrm{kPa}$ at the field intensity of $4 \mathrm{kV} / \mathrm{mm}$, as reported. ${ }^{14}$ Shearing tests have shown that the dispersion has a zero-field viscosity of $9.5 \mathrm{~Pa} \mathrm{~s}$, and field-dependent yield shear stress $\tau_{y}=1953 E^{1.39}$ (the units of $\tau_{y}$ and $E$ are Pa and $\mathrm{MV} / \mathrm{m}$, respectively).

The sample was tested on the instrument described above with two sizes, $\phi=25.4 \mathrm{~mm}$ and $\phi=50.8 \mathrm{~mm}$, of the

TABLE I. Test conditions.

\begin{tabular}{ccccccccrr}
\hline \hline Test No. & $\begin{array}{c}\text { Plate diam. } \\
(\mathrm{mm})\end{array}$ & $\begin{array}{c}\text { Initial gap } \\
(\mathrm{mm})\end{array}$ & $\begin{array}{c}\text { Voltage } \\
(\mathrm{V})\end{array}$ & $\begin{array}{c}p_{c} \\
(\mathrm{kPa})\end{array}$ & Test No. & $\begin{array}{c}\text { Plate diam. } \\
(\mathrm{mm})\end{array}$ & $\begin{array}{c}\text { Initial gap } \\
(\mathrm{mm})\end{array}$ & $\begin{array}{c}\text { Voltage } \\
(\mathrm{V})\end{array}$ & $\begin{array}{r}p_{c} \\
(\mathrm{kPa})\end{array}$ \\
\hline 1 & 50.8 & 0.6 & 0 & $\ldots$ & 12 & 25.4 & 0.6 & 1200 & 21.3 \\
2 & 50.8 & 0.6 & 600 & 16.2 & 13 & 25.4 & 0.6 & 1600 & 31.7 \\
3 & 50.8 & 1.0 & 1000 & 33.1 & 14 & 25.4 & 1.0 & 600 & 8.1 \\
4 & 50.8 & 1.0 & 2000 & 86.7 & 15 & 25.4 & 1.0 & 2000 & 43.3 \\
5 & 50.8 & 1.0 & 3000 & 152.3 & 16 & 25.4 & 1.0 & 2600 & 62.4 \\
6 & 50.8 & 2.0 & 1200 & 42.6 & 17 & 25.4 & 1.5 & 1000 & 16.5 \\
7 & 50.8 & 2.0 & 2000 & 86.7 & 18 & 25.4 & 1.5 & 1600 & 31.7 \\
8 & 50.8 & 2.0 & 4000 & 227.1 & 19 & 25.4 & 1.5 & 2600 & 62.4 \\
9 & 25.4 & 0.4 & 400 & 4.6 & 20 & 25.4 & 2.0 & 2000 & 43.3 \\
10 & 25.4 & 0.4 & 800 & 12.1 & 21 & 25.4 & 2.0 & 4000 & 113.6 \\
11 & 25.4 & 0.6 & 600 & 8.1 & 22 & 25.4 & 2.0 & 5000 & 154.8 \\
\hline \hline
\end{tabular}




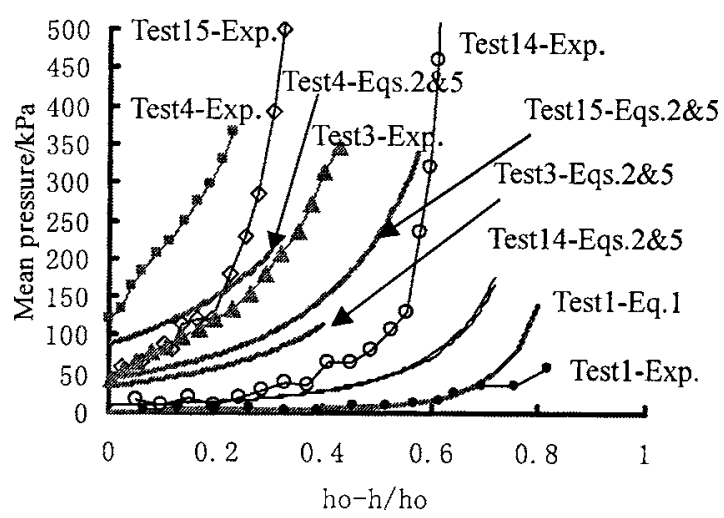

FIG. 2. Variation of pressure with reduction in electrode separation.

upper electrode, under different conditions of initial electrode gap and applied voltage as listed in Table I. A personal computer (PC) equipped with a 12 bit high sampling rate $A / D$ converter was utilized to acquire the outputs of the load cell, torque sensor, and displacement sensors A and B, and necessary filtering treatments were done on the digitized data.

In this paper, the results obtained in unidirectional compression experiments are presented. Here the unidirectional compression means that the upper electrode moves down continuously from a preset position to squeeze a dispersion sample in a voltage-constant electric field until the resistance force of the sample under testing reaches to a value near the capacity of the load cell. The speed of the moving head, referred to as nominal compression speed hereafter, was set to be a constant of $14.5 \mu \mathrm{m} / \mathrm{s}$ for test 1 and $7.25 \mu \mathrm{m} / \mathrm{s}$ for all of the other tests. It should be noted that the actual compression speed slowed down during the compression test because of the growing elastic deformation in the loading and supporting parts of the test device under high pressure. All of the experiment was performed at the room temperature of $22 \pm 1{ }^{\circ} \mathrm{C}$.

\section{RESULTS AND DISCUSSIONS}

Figure 2 shows a few typical experimental curves of the compressive resistance versus the fractional reduction in the electrode gap. In this figure, the compressive resistance of the dispersion sample is expressed in terms of mean pressure $p$, which is the measured compression force $F$ divided by the area of the upper electrode $S$. When no external voltage was applied, the dispersion sample showed very weak compressive resistance until the gap width decreased down to $0.1 \mathrm{~mm}$ or smaller. The mean pressure $p$, in this case, can be estimated from the theory of squeeze flow of viscous fluids,

$$
p=\frac{3 \pi \eta W r_{0}^{2}}{h^{3}(t)}
$$

where $\eta$ is the viscosity of the dispersion at the zero-field intensity, $W$ the compression velocity, $r_{0}$ the radius of the upper electrode and $h(t)$ the electrode gap width at any instant time $t$ during compression. For the sample tested, its zero-field viscosity $\eta$ was measured to be $9.5 \mathrm{~Pa}$ s and $W$ and $r_{0}$ were $14.5 \mu \mathrm{m} / \mathrm{s}$ and $25.4 \mathrm{~mm}$, respectively, in this case.
The mean pressure calculated by substituting these values into Eq. (1) are also plotted in the figure. The experimental data fairly follow the theory up to a large extent of compression. The discrepancy which appears at the large gap reductions is caused by the slowing down of the upper electrode, which is lower than the nominal compression speed of $14.5 \mu \mathrm{m} / \mathrm{s}$ (when the compression force gets large enough to produce significant elastic deformation on the polyoxymethylene insulating cylinder). The results manifest the fact that the viscous contribution to the total compression force is negligible in the ranges of compression velocity and electrode gap width used in the experimental study compared with the effect of electric field as described below.

When the electrorheological fluid sample was squeezed in a strong electric field, its compressive resistance increased greatly with the gap reduction as shown in the figure. In the experiments with the electrode of a smaller size, the mean pressure went up over $500 \mathrm{kPa}$, and the peak pressure value for the experiments with the electrode of a larger size was limited to about $350 \mathrm{kPa}$ because of the capacity of the load cell. In fact, in all of the experiments carried out in a relatively strong electric field, the compression force readily exceeded $800 \mathrm{~N}$ within a gap reduction of less than 0.6. The higher the field intensity was, the greater the mean pressure was at a given gap reduction and the more rapid the mean pressure increased with the gap reduction. For the same field intensity, the larger electrode size resulted in greater mean pressure than the smaller electrode size. It is clear that the compression resistance of the electrorheological fluid depends on the applied voltage, electrode gap reduction, and electrode size.

According to the squeeze-flow theory developed by Williams et al., the main pressure $p(t)$ of electrorheological fluids at any gap width $h(t)$ can be expressed by the following equation:

$$
p(t)=\frac{2 \tau_{y}\left[\frac{V}{h(t)}\right] r_{0}}{h(t) \chi^{3}}\left\{\frac{\gamma^{3}}{108}+\int_{\gamma / 3}^{\chi} G S d S\right\},
$$

where $\tau_{y}$ is the yield shear strength of the fluid, which is a function of the applied voltage $V$ and the gap width $h(t)$, taking the form of $\tau_{y}=A[V / h(t)]^{b}$. Here $A$ and $b$ are material constants and $\gamma, \chi, S$, and $G$ are parameters defined by

$$
\begin{aligned}
& \gamma=\frac{\eta}{\eta_{r}} ; \quad S=\frac{W \eta r}{h^{2}(t) \tau_{y}\left[\frac{V}{h(t)}\right]} ; \quad \chi=\left.S\right|_{r=r_{0}} ; \\
& G=-\frac{h(t)}{2 \tau_{y}\left[\frac{V}{h(t)}\right] \frac{d p}{d r}},
\end{aligned}
$$

respectively, where $\eta_{r}$ is the preyield viscosity in the biviscous model, $r$ the coordinate in the radial direction, and $p$ the pressure. ${ }^{5}$ When $S$ is in the range from $\gamma / 3$ to $\chi$, the dimensionless pressure gradient $G$ is defined in terms of $S$ and $\gamma$ by the following cubic equation:

$$
2 G^{3}-3[2 S-\gamma+1] G^{2}+1-\gamma=0 .
$$


Taking $\gamma=0.0001$ and using the curve-fit formula $\tau_{y}$ $=1953[V / h(t)]^{1.39}$ (the units of $\tau_{y}, V$, and $h(t)$ are Pa, MV, and $\mathrm{m}$, respectively) obtained by shear tests, we calculated the mean pressure for these tests by numerically solving Eq. (3) and integrating Eq. (2). The calculated results were presented as solid lines in Fig. 2. We can see that the theory underestimates the compressive resistance, especially when the reduction is large.

When plasticity number $S$ is less than 0.5 , pressure gradient $G$ and thus the compression force are predominated by the yield shear stress, and viscous effect has little contribution (see Fig. 4 in Ref. 5). Under this condition, the complicated expression (2) can be simplified by setting $\gamma=0$ as

$$
F(t)=\frac{2 \pi r_{0}^{3}}{3 h(t)} \tau_{y}\left[\frac{V}{h(t)}\right],
$$

which means that the dispersion material behaves like an ideal plastic medium under compression. And thus the mean pressure $p$ is given by

$$
p=\frac{2 r_{0}}{3 h(t)} \tau_{y}\left[\frac{V}{h(t)}\right] .
$$

In comparison, the curves calculated from Eq. (5) for the above test cases are also plotted as dashed lines in Fig. 2 . The dashed lines locate slightly below the corresponding solid lines, and the difference between them is less than $3 \%$. In other words, the Williams's squeeze-flow theory has the simple solution of Eq. (5), in these situations. In all of the compression tests in the electric fields discussed in this paper, plasticity number $S$ is estimated to be less than 0.5 , and thus Eq. (5) is applicable.

By introducing the relationship of $\tau_{y}=A(V / h)^{b}$, we can rewrite Eq. (5) as

$$
\frac{p}{p_{c}}=\left(\frac{h_{c}}{h(t)}\right)^{1+b},
$$

or in the natural logarithmic form as

$$
\ln p=\ln p_{c}+(1+b) \ln h_{c}-(1+b) \ln h(t),
$$

in which

$$
p_{c}=\frac{2 r_{0}}{3 h_{c}} \tau_{y}\left(\frac{V}{h_{c}}\right)=\frac{2 r_{0}}{3 h_{c}} A\left(\frac{V}{h_{c}}\right)^{b},
$$

and $h_{c}$ is a specific electrode gap width which can take an arbitrary value. Taking $h_{c}=1 \mathrm{~mm}$, then Eq. (7) becomes

$$
\ln p=\ln p_{c}-(1+b) \ln h(t) .
$$

Equation (9) means that in a ln-ln plot the mean pressure $p$ changes with the electrode gap $h(t)$ linearly with the slope of $(1+b)$ and the intercept $\ln p_{c}$. For a given electrorheological fluid, $b$ is a material constant, and hence $\ln p$ versus $\ln h(t)$ lines have the same slope of $(1+b)$, regardless of electrode plate size, applied voltage, and material constant $A$. Besides, initial electrode gap width affects neither the slope nor the intercept according to the theory. The characteristic parameter $p_{c}$, calculated from Eq. (8) for each test, is listed

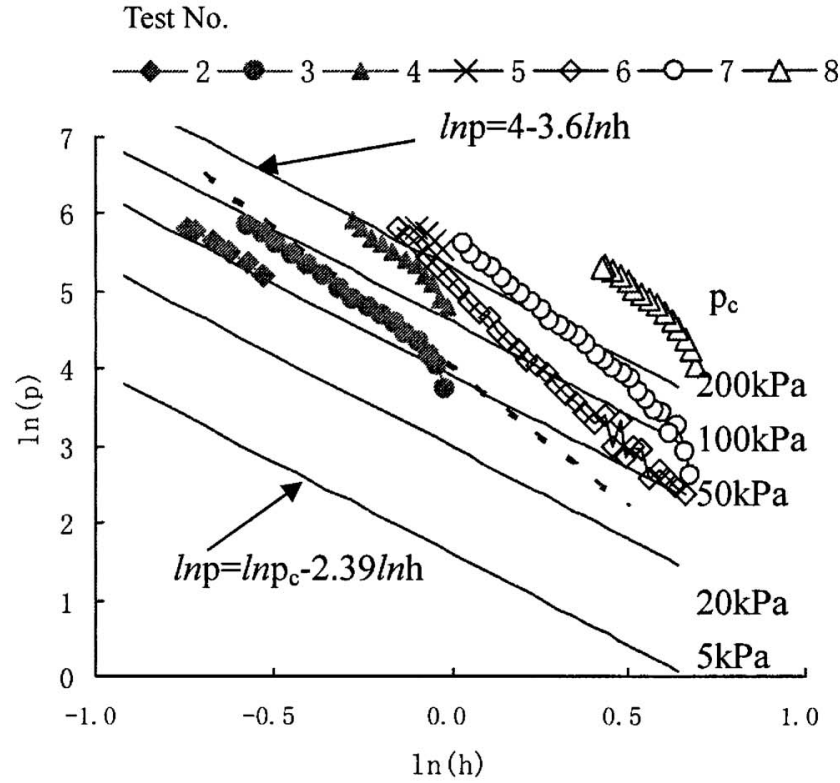

(a) Plate diameter $\mathrm{D}=50.8 \mathrm{~mm}$
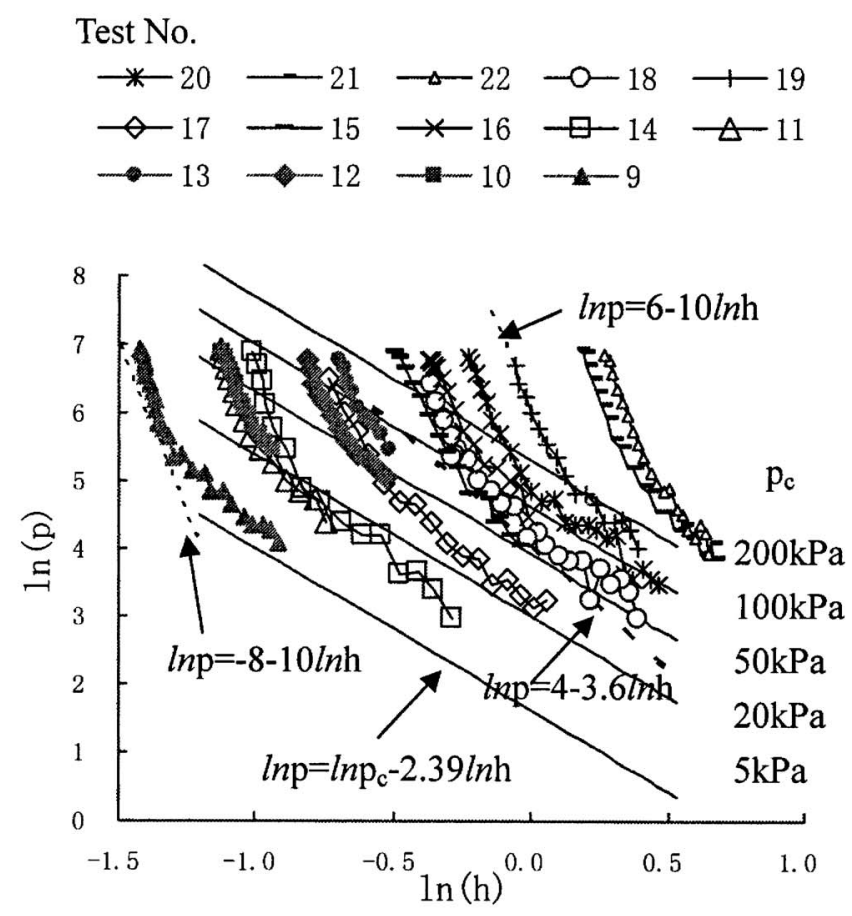

(b) Plate diameter $\mathrm{D}=25.4 \mathrm{~mm}$

FIG. 3. Variations of pressure $p$ with the electrode separation $h$ in various conditions: (a) Plate diameter $d=50.8 \mathrm{~mm}$ and (b) plate diameter $d$ $=25.4 \mathrm{~mm}$.

in the last column in Table I. In the calculation, $h_{c}$ was set as $1.0 \mathrm{~mm}$ for all of the tests, and the material constants of $A$ and $b$ were $1953 \mathrm{~Pa}$ and 1.39 as mentioned above.

The experimental $\ln p$ versus $\ln h(t)$ curves are classified into two groups, according to the electrode plate size, and displayed in Figs. 3(a) and 3(b), respectively, together with the prediction lines calculated from Eq. (9) with several typical $p_{c}$ values and $b=1.39$. In the case of the larger electrode plate, all seven experimental curves look like straight lines, 
excluding at the right end where the pressure goes down suddenly due to the transition between elastic deformation and plastic flow.

The slopes of the experimental curves, however, do not accord with the theoretical one, 2.39, but differ with each other, varying in the range from 2.9 to 4.2 with the average value of 3.6. It seems that the slopes are dependent on neither the applied voltage nor the initial electrode separation. The $\pm 16 \%$ discrepancy between the slopes of the seven test curves is probably caused by the scatter of the dispersion's rheological properties as well as measurement errors. In comparison, a dash line with the slope of 3.6 was also added in the figure. It should be noted that the nonlinear conduction model $^{15}$ of electrorheological effect predicts a nonparabolic $(V / h)^{3 / 2}$ dependence of the yield shear stress $\tau$, i.e., $b=1.5$.

The fact that most of the test results show a slope greater than 2.39 implies that the compression resistance of the electrorheological fluid increases with the decrease of electrode separation more rapidly than the prediction of the squeezeflow theory. Besides the slope, the theory also underestimates the compression resistance at a given electrode gap width $h$. This point can be seen by comparing a test curve with the prediction lines whose intercept parameter $p_{c}$ is near to that of the test listed in Table I. For instance, the test numbered 3 takes the $p_{c}$ of $33.1 \mathrm{kPa}$. According to Eq. (8), the test curve is beyond the prediction line of $p_{c}=50 \mathrm{kPa}$. If we focus the pressure on the right end of each test which corresponds to the beginning of compression, we can find that the discrepancy between the theory and experiment is greater in the case of the smaller initial gap widths than in the case of the larger initial gaps. It is also greater for the higher voltage applications than for the lower voltage applications when the initial gap width is the same. This indicates that the continuum squeeze-flow theory describes the compression behavior of electrorheological fluids poorly in the conditions of small initial gaps and high voltages, being consistent with the experimental results reported by Tian et al. ${ }^{13}$

In the case of the smaller plate size, compression can go further within the capacity of the load cell used than in the larger plate case. From Fig. 3(b), we can see that with the increase of the gap reduction the measured pressure goes up at the approximately same rate as that in the larger plate case (seeing the dash line of $\ln p=4-3.6 \ln h$ ) in the early stage. This gradually turns up at a much higher rate. The slope of the test curves in the last stage of compression and reaches to approximately 10 , as shown with the two thin dash lines in the figure. All of the 14 tests with different initial gap and voltage settings show similar tendencies. In the same way as in the larger plate case, initial gap and voltage settings have little influence on the variation pattern and the slopes of the test curves, but affect the characteristic parameter of $p_{c}$.

The over-logarithmic relationship between the compression resistance and the electrode gap cannot be described by a homogenous non-Newtonian model as assumed in the William's squeeze-flow theory. To explain such a behavior of electrorheological fluids, evolution of particle aggregation during compression needs to be considered. According to the experimental finding reported in Ref. 4, before a shear field is applied, particles in the dispersion assemble together into numerous columnarlike clusters in the presence of an electric field, and the diameter of the clusters is in the range of 100-200 $\mu \mathrm{m}$. Assuming that the diameter $d$ of the fieldinduced particle clusters is the same for a given field intensity before compressed, the aspect ratio $h / d$ of the particle clusters is different for different electrode separation $h$. In the beginning of compression, the aspect ratio $h / d$ is determined by the initial electrode separation $h_{0}$. If $h_{0}$ is relatively large, saying $2.0 \mathrm{~mm}$ or greater, the aspect ratio $h_{0} / d$ could be about or higher than 10 and the particle clusters could collapse under compression and be squeezed out together with oil. The continuum non-Newtonian squeeze-flow theory can be applicable, with some extent of accuracy, to describe the behavior at the very beginning stage of compression. If $h_{0}$ is relatively small, saying $0.6 \mathrm{~mm}$ or less, the aspect ratio could be lower than 5, resulting in higher stability of the particle clusters and thus higher compression resistance.

With the progress in compression, the electrode gap separation $h$ decreases. Meanwhile, the cluster diameter $d$ could increase gradually because the existence of electric field inhibits particles from escaping out of the gap and the clusters bind with each other along with the loss of oil between them. Finally, one or more consolidated particle clusters with certain critical low aspect ratio could form in the electrode gap. Once the stumpy consolidated clusters appear the compression mode changes to that similar to the compaction of granular materials, and the resistance thus increases greatly. Meanwhile, the change in microstructure can result in a great increase of shear resistance too, as reported by Tao et al. ${ }^{11}$

\section{CONCLUSIONS}

Electrorheological fluids are regarded as Bingham-like non-Newtonian fluids. Continuum non-Newtonian squeezeflow theory is often used to describe the response of the fluids which are subjected to oscillatory or unidirectional compression. The study gives an extension of the theory for quasistatic compression through normalization of the compression pressure. The experimental results obtained with different electrode sizes, initial electrode separations, and applied voltages in this study show that the theory fails to accurately predict the compression resistance of the fluids throughout the compression process. Moreover, it is found that the mean pressure increases with the progress in compression at a much higher rate than the theoretical prediction in the presence of a strong electric field. When the initial electrode separation is down to submillimeters, the theory underestimates the compression resistance even at the beginning of compression. An analysis of morphological change in particle clusters under compression has been given to explain the compression behavior.

\section{ACKNOWLEDGMENTS}

One of the authors (Y.M.) is grateful for the support from the China Scholarship Council for his visiting research at the University of Michigan. The grants from the National Basic Research Program of China (No. 2003CB716205) and NSFC are acknowledged. 
${ }^{1}$ W. M. Winslow, J. Appl. Phys. 21, 1137 (1949).

${ }^{2}$ Y. Tian, Y. Meng, and S. Wen, Phys. Rev. E 65, 031507 (2002).

${ }^{3}$ R. Tao and Q. Jiang, in Progress in Electrorheology, edited by F. E. Filisko and K. Havelka (Plenum, New York, 1995), pp. 325-333.

${ }^{4}$ S. Henley and F. E. Filisko, J. Rheol. 45, 1323 (1999).

${ }^{5}$ E. W. Williams, S. G. Rigby, J. L. Sproston, and R. Stanway, J. NonNewtonian Fluid Mech. 47, 221 (1993).

${ }^{6}$ H. See, J. S. Field, and B. Pfister, J. Non-Newtonian Fluid Mech. 84, 149 (1999).

${ }^{7}$ M. Nilsson and N. Ohlson, J. Intell. Mater. Syst. Struct. 11, 545 (2000).
${ }^{8}$ G. J. Monkman, J. Phys. D 28, 588 (1995).

${ }^{9}$ H. Q. Gong and M. K. Lim, J. Intell. Mater. Syst. Struct. 7, 89 (1996).

${ }^{10}$ S. L. Vieira, M. Nakano, R. Oke, and T. Nagata, Int. J. Mod. Phys. B 15, 714 (2001).

${ }^{11}$ R. Tao, Y. C. Lan, and X. Xu, Int. J. Mod. Phys. B 16, 2622 (2002).

${ }^{12}$ X. Tang, X. Zhang, and R. Tao, J. Appl. Phys. 87, 2634 (2000).

${ }^{13}$ Y. Tian, S. Wen, and Y. Meng, Phys. Rev. E 67, 051501 (2003).

${ }^{14}$ Y. Tian, Y. Meng, and S. Wen, J. Appl. Phys. 90, 493 (2001).

${ }^{15}$ P. Atten, J.-N. Foulc, and N. Filici, Int. J. Mod. Phys. B 8, 2731 (1994). 